\title{
Atypical/Nor98 scrapie: properties of the agent, genetics, and epidemiology
}

\author{
Sylvie L. BENESTAD ${ }^{1 *}$, Jean-Noël ARSAC ${ }^{2}$, Wilfred GOLDMANN ${ }^{3}$, \\ Maria NÖREMARK ${ }^{4}$ \\ ${ }^{1}$ National Veterinary Institute, PO Box 8156 Dep., Oslo, Norway \\ ${ }^{2}$ Agence Française de Sécurité Sanitaire des Aliments, 31 av. Tony Garnier, 69364 Lyon Cedex 07, France \\ ${ }^{3}$ Neuropathogenesis Unit, Roslin Institute, Ogston Bldg, West Mains Rd, Edinburgh, EH9 3JF, UK \\ ${ }^{4}$ National Veterinary Institute, 75189 Uppsala, Sweden
}

(Received 23 July 2007; accepted 23 October 2007)

\begin{abstract}
Atypical/Nor98 scrapie cases in sheep were diagnosed for the first time in Norway in 1998. They are now identified in small ruminants in most European countries and represent an increasingly large proportion of the scrapie cases diagnosed in Europe. Atypical/Nor98 scrapie isolates have shown to be experimentally transmissible into transgenic mice and sheep but the properties of the TSE agent involved, like its biological and biochemical features, are so clearly distinct from the agent involved in classical scrapie that they have provided a challenging diagnostic for many years. No strain diversity has yet been identified among the atypical/Nor98 scrapie sample cases. The genetic predisposition of the sheep affected by atypical/Nor98 scrapie is almost inverted compared to classical scrapie, and the exact origin of this sporadic TSE strain is still speculative, but a spontaneous, non-contagious origin, like sporadic CreutzfeldtJakob disease in humans, can not be excluded. Further transmission and epidemiological studies are needed to better address this hypothesis.
\end{abstract}

atypical scrapie / Nor98 / transmissible spongiform encephalopathies TSE / genetics / epidemiology

Table of contents

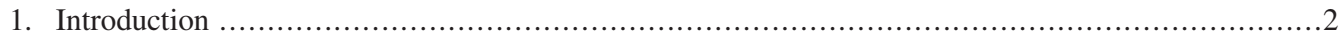

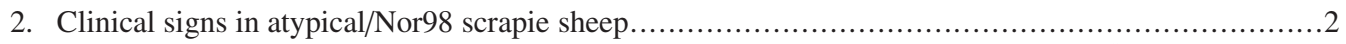

3. Atypical/Nor98 scrapie brains have a different appearance and distribution of the $\mathrm{PrP}^{\mathrm{Sc}}$ compared to classical scrapie as revealed by immunohistochemistry ..............................................

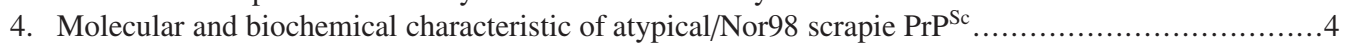

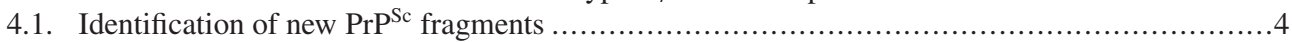

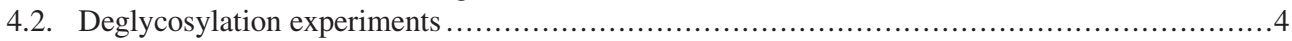

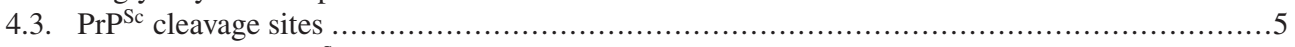

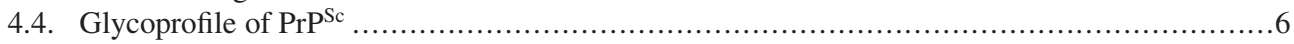

4.5. Proteinase $\mathrm{K}$ resistance of the $\mathrm{PrP}^{\mathrm{Sc}}$ in atypical/Nor98 scrapie isolates........................... 7

5. Absence of detectable lymphotropism in atypical/Nor98 scrapie cases ............................. 7

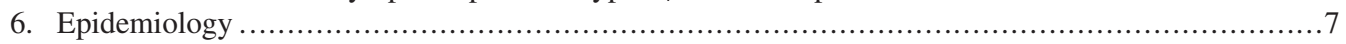

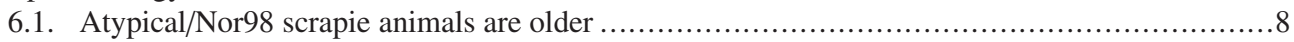

6.2. Prevalence and factors affecting the ability to detect atypical/Nor 98 scrapie cases ............... 8

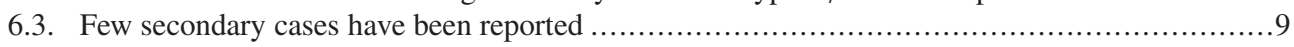

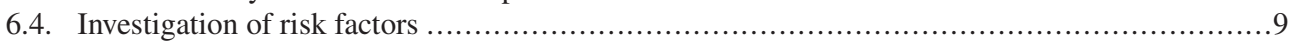

*Corresponding author: sylvie.benestad@ vetinst.no 


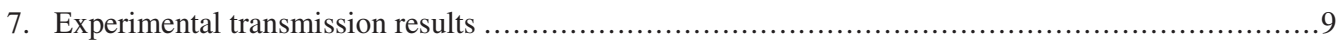

7.1. Failure of experimental transmission to standard mice...........................................

7.2. Successful experimental transmission to transgenic mice ...................................... 10

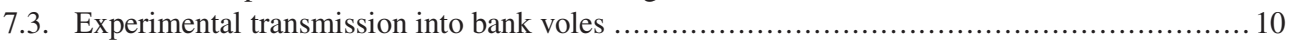

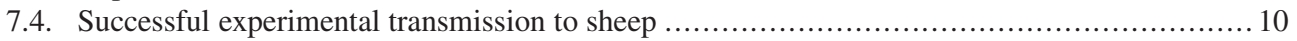

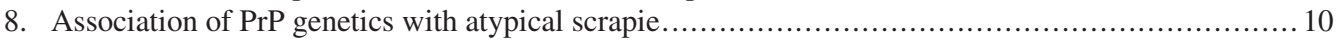

9. Bio-diversity or uniformity of atypical/Nor98 scrapie isolates? ................................. 11

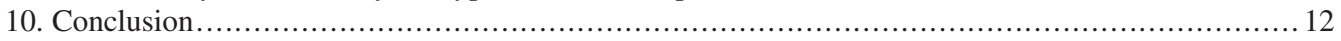

\section{INTRODUCTION}

Prion diseases, also called transmissible spongiform encephalopathies (TSE), are a group of fatal neurodegenerative diseases affecting, among others, humans (Creutzfeldt-Jakob disease and GerstmannSträussler-Scheinker disease (GSS)), cattle (bovine spongiform encephalopathies (BSE)), and small ruminants (scrapie). The precise nature of the causative agent is still not known, but these diseases are tightly associated with the accumulation in infected tissues of a misfolded, partially proteinase-K resistant isoform $\left(\mathrm{PrP}^{\mathrm{Sc}}\right)$ of the cellular prion protein $\left(\mathrm{PrP}^{\mathrm{C}}\right)$ [34], and $\mathrm{PrP}^{\mathrm{Sc}}$ is therefore used as a diagnostic marker for the disease.

Scrapie in sheep has been described in several European countries for over two centuries [39] and is the prototype of the prion diseases. Experimental transmission studies have shown the biological diversity of the scrapie agents, characterised after sub passages in mice by distinct incubation periods and distribution of the brain lesions, defining different strains. In 1998, an unusual type of scrapie was diagnosed in Norwegian sheep and was subsequently designated Nor98 scrapie [5]. Since the implementation in Europe of a large surveillance program for TSE in small ruminants, an increasing number of cases of atypical/Nor98 scrapie $^{1}$ has been reported in

\footnotetext{
${ }^{1}$ An unusual type of scrapie was identified for the first time in Norway in sheep in 1998 and was therefore designated as scrapie Nor98. Since the publication of the discovery, atypical scrapie cases have been diagnosed in most of the European countries in sheep and goat populations. The vast majority of these atypical cases are similar if not identical to Nor98 cases, but they are designated outside Norway as "Nor98", "Nor98-like", or "atypical scrapie". The authors therefore choose to
}

the majority of the European countries [9, 13, $15,16,31-33,37]$, with also one case in the Falkland Islands [14] and in the USA [12]. A case was recently retrospectively found in UK archive material dating from 1989 [7].

In 2005, the European Food Safety Authority (EFSA) published a classification of TSE in small ruminants ${ }^{2}$, dividing them into three diagnostic categories: classical scrapie, BSE in small ruminants, and atypical scrapie including Nor98, as illustrated in Table I.

TSE strains are defined as infectious isolates that exhibit distinct biological and biochemical features and that cause, upon inoculation, prion disease with consistent characteristics like their host spectrum, incubation periods, neuropathological changes, courses of the disease and clinical signs, and the biochemical characteristics of the corresponding $\mathrm{PrP}^{\mathrm{Sc}}$. It is thought that the information necessary to determine a strain is dependant on the conformation of the $\mathrm{PrP}^{\mathrm{Sc}}$ (reviewed in [1]).

The present paper will review what is known about the characteristics of the atypical/Nor98 scrapie cases.

\section{CLINICAL SIGNS IN ATYPICAL/NOR98 SCRAPIE SHEEP}

A large majority of the atypical/Nor98 scrapie sheep have been diagnosed through

use the term "atypical/Nor98 scrapie" in the present review.

${ }^{2}$ EFSA: Opinion of the scientific panel on biological hazards on the request from the European Commission on classification of atypical transmissible spongiform encephalopathy (TSE) cases in small ruminants. EFSA J. (2005) 276:1-30. http://www.efsa.europa.eu/EFSA/efsa_locale1178620753812_1178620776235.htm [consulted 23 October 2007]. 
Table I. Categorisation of TSE in small ruminants. The table is adapted from the EFSA opinion $(2005)^{2}$.

\begin{tabular}{|c|c|c|c|c|}
\hline TSE type & $\begin{array}{l}\text { "Stringent" } \\
\text { Western blot }\end{array}$ & $\begin{array}{l}\text { "Mild- } \\
\text { proteinase } \\
\text { K"Western blot }\end{array}$ & $\begin{array}{l}\text { Immunohistochemistry } \\
\text { and histopathology }\end{array}$ & Comments \\
\hline $\begin{array}{l}\text { Classical } \\
\text { scrapie }\end{array}$ & $\begin{array}{l}\text { Three band } \\
\text { pattern above } \\
15 \mathrm{kDa} \text {. }\end{array}$ & $\begin{array}{l}\text { Three band } \\
\text { pattern above } \\
15 \mathrm{kDa} \text {. }\end{array}$ & $\begin{array}{l}\text { Grey matter vacuolation } \\
\text { immuno-labelling in the } \\
\text { medulla involving the } \\
\text { DMNV. }\end{array}$ & $\begin{array}{l}\text { Susceptible genotypes } 2- \\
5 \text { years old. }\end{array}$ \\
\hline $\begin{array}{l}\text { BSE in small } \\
\text { ruminants }\end{array}$ & $\begin{array}{l}\text { Three band pattern. } \\
\text { the di-glycosylated ba } \\
\text { staining with mAb } \\
\text { the N-terminus. Ungly } \\
\text { lower than in classical }\end{array}$ & $\begin{array}{l}\text { Dominance of } \\
\text { and. No or weak } \\
\text { raised against } \\
\text { lycosylated band } \\
\text { 1 scrapie. }\end{array}$ & $\begin{array}{l}\text { Grey matter vacuolation } \\
\text { immuno-labelling in the } \\
\text { medulla involving the } \\
\text { DMNV. }\end{array}$ & \\
\hline Atypical/Nor98 & $\begin{array}{l}\text { Negative or show- } \\
\text { ing a Western blot } \\
\text { profile distinct from } \\
\text { other TSE. }\end{array}$ & $\begin{array}{l}\text { Multiple band } \\
\text { pattern with a } \\
\text { lower band at } \\
\text { below } 15 \mathrm{kDa} \text {. }\end{array}$ & $\begin{array}{l}\text { No involvement of the } \\
\text { DMNV }{ }^{1} \text {. Immunostaining } \\
\text { often present in the nucleus } \\
\text { of the spinal tract of the } \\
\text { trigeminal nerve. Immunos- } \\
\text { taining generally more } \\
\text { intense in the cerebellar and } \\
\text { cerebral cortices. }\end{array}$ & $\begin{array}{l}\text { Commonly older sheep } \\
\text { (4 years and more). Geno- } \\
\text { types associated with } \\
\text { resistance to classical } \\
\text { scrapie. Preferences for } \\
\text { the AHQ, } \mathrm{AF}_{141} \mathrm{RQ} \text {, and } \\
\mathrm{ARR} \text { alleles. Most often } \\
\text { single cases per flock. }\end{array}$ \\
\hline
\end{tabular}

DMNV: dorsal motor nucleus of the vagus.

an active surveillance in apparently healthy sheep or fallen stock animals and not as clinical suspect cases. This might suggest that the clinical signs, if present, can be different or less pronounced than the signs observed for classical scrapie, but could also be a result of the epidemiology where often only single cases are detected in a flock and farmers might not contact a veterinarian when only one animal is showing signs of disease. When cases are detected through active surveillance the data available on clinical signs, or absence of such, is many times restricted to what might have been observed by the farmer. There are a limited number of cases described to be detected through clinical suspicion where, more detailed information from veterinary clinical examinations is available [23].

The salient clinical sign reported in the Nor98 affected animals in Norway is ataxia. Other signs were anxiety and loss of body condition [5, 6]. Hind limb ataxia was also reported in two Swedish cases, one of them was also described as stiff and with poor body condition, while other Swedish cases had not displayed any clinical symptoms according to the farmer [16]. Two clinical suspect cases reported in Ireland shared the same clinical sign, incoordination, in addition to weight loss and nervous behaviour [32]. The case in the Falkland Islands displayed poor body condition and an abnormal behaviour with persistent biting at its leg and had shown collapsing episodes when it tried to run [14]. The two cases reported in the UK [23] were also ataxic and showed delayed repositioning of the hind limbs. It was noted that both displayed signs suggestive of a cerebellar disease but the signs were not identical (hypermetria in the first case, ataxia, fine head tremor, and abnormal menace response in the second case). In addition, the first case exhibited nervousness while the second case showed dullness, and it was speculated in this report that these differences might be due to different clinical durations, as supported by the higher level of $\mathrm{PrP}^{\mathrm{Sc}}$ found by Western blot in the brain of the second case.

Pruritus has not been reported in the atypical/Nor98 scrapie cases, but one of the cases detected on clinical suspicion in the UK showed a positive scratch response [23]. 


\section{ATYPICAL/NOR98 SCRAPIE BRAINS HAVE A DIFFERENT APPEARANCE AND DISTRIBUTION OF THE PRP $^{\text {SC }}$ COMPARED TO CLASSICAL SCRAPIE AS REVEALED BY IMMUNOHISTOCHEMISTRY}

In the atypical/Nor98 scrapie cases, the $\mathrm{PrP}^{\mathrm{Sc}}$ deposition type appears as fine punctate to coarse granular deposits observed both in the grey and white matters when examined by immunohistochemistry. No intraneuronal staining has ever been reported, contrasting to most of the classical scrapie isolates.

In the brainstem, some cases have been described as totally negative at the level of the obex $[5,9,10,16,32]$ while other cases display minimal staining restricted only to the spinal tract nucleus of the trigeminal nerve [5, $31,33,37,41]$. The primary structure affected in classical scrapie, the dorsal motor nucleus of the vagus (DMNV) was never affected. A particularly heavy and pronounced globular staining of the white matter tracts has in some cases been observed as the only staining at the level of the obex, or in addition to the spinal tract nucleus of the trigeminal nerve, and sometimes to the reticular formation and the ambiguus nucleus [31]. In general, the intensity of $\mathrm{PrP}^{\mathrm{Sc}}$ immunostaining observed in the medulla oblongata is generally much less in the atypical/Nor98 scrapie cases compared to the classical scrapie cases. On the contrary to classical scrapie, the cerebellar and cerebral cortices are generally the most immunostained areas of the atypical/Nor98 scrapie brains. But in these areas too, small differences are noticeable. A diffuse fine punctate or alternatively a patchy staining (never stellate), in some cases very discrete while conspicuous in other cases, is reported in the molecular and granular layers and in the white matter, or in two or even in only one of these three structures $[5,9,10,16$, $31,32,37]$. It is important to underline that immunostaining can be minimal or totally absent from the cerebellum in some atypical/Nor98 scrapie cases while in other cases the only brain area showing immunostaining is in the cerebellum $^{3}[31]$.

\footnotetext{
${ }^{3}$ Benestad, unpublished results.
}

Another characteristic has been reported in some atypical/Nor98 scrapie cases where the only anatomical brain region showing a type of perineuronal and linear staining similar to what is observed in classical scrapie (see [17]) has been the substantia nigra (Fig. 1A). Of significance is also the presence of plaque-like, dense focal or multifocal aggregates found mostly in the tectum and in the lateral geniculate body of the midbrain, and the white matter tracts of the cerebral cortex (Fig. 1B).

\section{MOLECULAR AND BIOCHEMICAL CHARACTERISTIC OF ATYPICAL/NOR98 SCRAPIE PRPSC}

\subsection{Identification of new $\operatorname{PrP}^{S c}$ fragments}

Up to 1998, all the scrapie strains classically showed, after proteinase $\mathrm{K}$ treatment and Western blot, a typical triplet pattern comprising the typical di-, mono-, and unglycosylated band migrating between 18 and $30 \mathrm{kDa}[19,20]$. Recently, three published biological studies have given a more precise description of newly identified fragments. The atypical/Nor98 scrapie isolates display after proteinase $\mathrm{K}$ treatment a distinct multiple band pattern, with a fast migrating band reported to be around $11-12 \mathrm{kDa}[2,5,18]$ or $7-8 \mathrm{kDa}[24,31]$ in size. These differences between the atypical/Nor98 scrapie and the classical scrapie isolates are illustrated in Figure 2.

It is noteworthy that, in contrast to what has been reported in human TSE, the same multiple band Western blot profile is observed independently of the brain region $[2,31]$.

\subsection{Deglycosylation experiments}

The molecular basis for these glycosylation differences between TSE strains is poorly understood. The fundamental mechanisms and their relation to disease characteristics are still unknown. To investigate the glycosylation of an isolate and to assess a Western blot profile, a PNGase enzyme (Peptide: N-glycosidase) can be used to cleave the carbohydrates from the protein and leave clear unglycosylated band(s) on the gel. A recent study [2] has investigated six atypical scrapie isolates from France and 


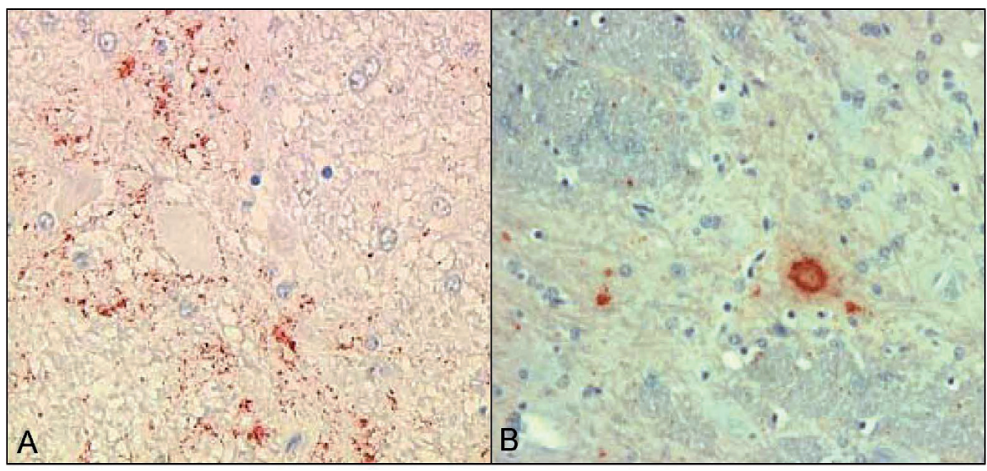

Figure 1. $\mathrm{PrP}^{\mathrm{Sc}}$ immunohistochemistry staining using F89/160.1.5 mAb antibody in two cases of atypical/Nor98 scrapie. A: Perineuronal and neuropil deposits $\mathrm{PrP}^{\mathrm{Sc}}$ in the substantia nigra; note the absence of intraneuronal staining. B: Plaque-like aggregates in the cerebral cortex (a color version is available on www.vetres.org).

one Nor98 isolate from Norway by Western blot. These isolates showed a similar pattern with five bands named band I to $\mathrm{V}$ with apparent molecular weights of around 31,27 , 21,18 , and $11 \mathrm{kDa}$ respectively, as illustrated in Figure 2. When the same isolates are deglycosylated with PNGase before Western blot analysis, the profile shows three bands referred to as bands A, B, and C migrating at around 23, 18 , and $11 \mathrm{kDa}$. This contrasts with the pattern observed in the classical isolate where the three band pattern $(27,23$, and $19 \mathrm{kDa}$ respectively) is transformed into a mono-band migrating at around $19 \mathrm{kDa}$ after deglycosylation.

Furthermore, Arsac et al. [2] calculated the theoretical molecular weights of monoglycosylated and bi-glycosylated forms potentially derived from $\mathrm{A}, \mathrm{B}$, and $\mathrm{C}$ fragments. They concluded that the A fragment $(23 \mathrm{kDa})$ and $\mathrm{B}$ fragment were present most probably also in their di- and mono-glycosylated forms as illustrated on Figure 2. Glycosylation of the A fragment would result in bands at $27 \mathrm{kDa}$ (band II) and $31 \mathrm{kDa}$ (band I) and that of the B fragment in bands at 21 (band III) and $27 \mathrm{kDa}$ (band II). Fragment C (11 kDa) was supposed to be only unglycosylated because the absence of detectable bands at $\cong 15$ and $\cong 19 \mathrm{kDa}$.

It is noticeable that the apparent molecular weight of the fragments of $\mathrm{PrP}^{\mathrm{Sc}}$ is different in the two molecular published studies:
Klingeborn et al. [24] used harsher proteinase $\mathrm{K}$ conditions, which, together with the applied antibodies, might explain at least partially the differences between the two studies. One of the isolates of the Swedish isolate has been analysed with the method used by Arsac et al. [2] (TeSeE Western blot, Bio-Rad), giving in these conditions a Western blot profile which was identical to the Norwegian Nor98 isolates. It is therefore highly plausible that the Nor98PrP7 fragment is equivalent to band $\mathrm{C}$ and the PrP-CTF14 is equivalent to band B.

\section{3. $\operatorname{PrP}^{\mathrm{Sc}}$ cleavage sites}

An additional feature of $\operatorname{PrP}^{\mathrm{Sc}}$ can be established using Western blotting by epitope mapping. Monoclonal antibodies directed against epitopes located directly or at the vicinity of the proteinase $\mathrm{K}$ cleavage site of the $\mathrm{PrP}^{\mathrm{Sc}}$ fragments are used. Multiple proteinase $\mathrm{K}$ digestion sites at the $\mathrm{N}$-terminus of the human, bovine, and ovine PrP have been identified this way. This variability in cleavage sites is thought to result from individual conformations of the N-termini of the protein that, by conferring varying three-dimensional structures to a single protein, lead to the exposure of distinct cleavage sites.

Three recent studies have contributed to new information concerning the cleavage sites of the atypical/Nor98 scrapie $\operatorname{PrP}^{\mathrm{Sc}}[2,18,24]$. 


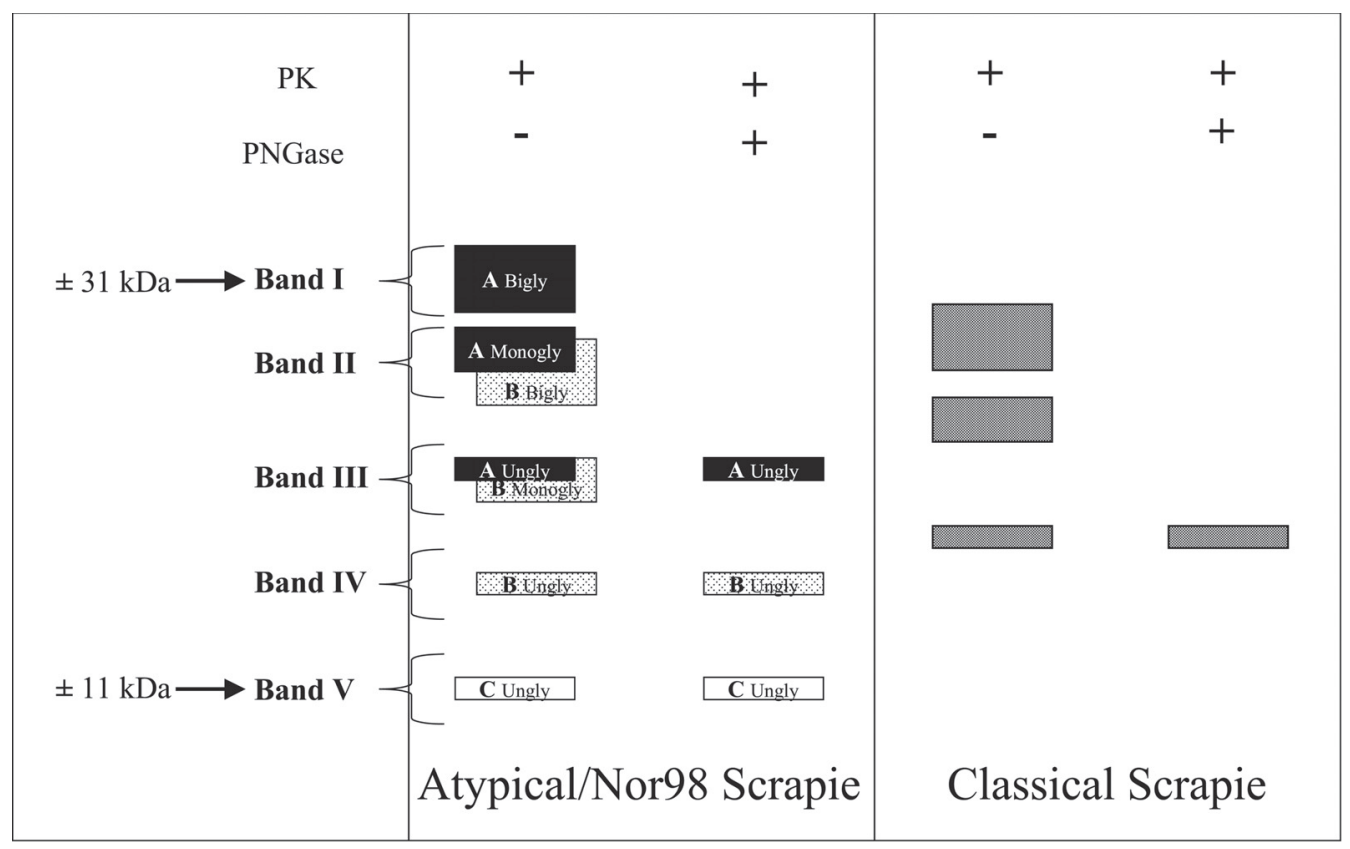

Figure 2. Schematic representation of the atypical/Nor98 scrapie $\mathrm{PrP}^{\mathrm{Sc}}$, adapted from the results from [2], showing the Western blot glycoprofile after proteinase K treatment of atypical/Nor98 scrapie using TeSeE ${ }^{\mathrm{TM}}$ Western blot (Bio-Rad), without and with removal of the carbohydrates by PNGase treatment. The five band pattern of the Nor98 isolates is replaced by a three band pattern after PNGase treatment. As a comparison, the classical scrapie isolate displays the typical three band pattern, replaced by one band after deglycosylation.

Taken together, their results show, as illustrated in Figure 3, that:

(i) Fragment A might correspond to a native (uncleaved or marginally cleaved by proteinase $\mathrm{K}$ treatment) PrP fragment.

(ii) Fragment B could be N-terminally ( aa 120 ) and C-terminally ( $\sim$ aa 233) cleaved.

(iii) The smallest fragment, called fragment $\mathrm{C}$ by Arsac et al. [2] and PrP7 by Klingeborn et al. [24], could correspond to an $\mathrm{N}(\sim$ aa 85$)$ and $\mathrm{C}(\sim$ aa $148-155)$ terminally truncated PrPres protein $(\sim$ aa 90 and $\sim$ aa 153 for Gretzschel et al. [18]) as estimated based on the mAb-binding sites retained. It is speculated that the truncation of the $\mathrm{C}$-terminus might occur in vivo, since the $\mathrm{C}$ fragment is not detectable in non-proteinase $\mathrm{K}$ treated isolates when la- belled with antibodies raised against the sequence situated upstream of aa 153 [18].

\subsection{Glycoprofile of $\operatorname{PrP}^{\mathrm{Sc}}$}

On immunoblots, characteristic differences can be observed based on the different intensities of the nonglycosylated, the mono-, and the diglycosylated $\mathrm{PrP}^{\mathrm{Sc}}$ bands. In some cases, such differences are remarkably robust and are widely used as indicators for prion strain typing $[11,20,38]$. Nevertheless, due to the lack of use of common standardised diagnostic tools between different TSE laboratories, it is today difficult to give a consistent description of the relative ratios of the multiple bands of $\mathrm{PrP}^{\mathrm{Sc}}$ from the atypical/Nor98 scrapie isolates. For example, the percentage of the total intensity represented by the lowest band that characterises the atypical/Nor98 scrapie cases varies considerably according to the mAb used and 
the proteinase $\mathrm{K}$ treatment of the isolate as illustrated by the differences observed in the literature $[2,5,9,24]$. It is quite plausible that most of the discrepancies could disappear by using common standardised diagnostic tools.

\subsection{Proteinase $K$ resistance of the $\operatorname{Pr} P^{S c}$ in atypical/Nor98 scrapie isolates}

It is a well-established fact that different strains differ significantly in their resistance to proteolytic degradation. Atypical/Nor98 scrapie isolates have been found to be much less resistant to proteinase $\mathrm{K}$ compared to classical scrapie [9]. This characteristic is believed to be at least partially responsible for the discrepancies observed between the test results obtained with different diagnostic tests [9], tests using more "stringent" proteinase K conditions having problems to identify atypical/Nor98 scrapie cases (Tab. I).

Klingeborn et al. [24], have demonstrated that especially the two smallest fragments of the atypical/Nor98 scrapie $\mathrm{PrP}^{\mathrm{Sc}}$, designated PrP7 and PrPCTF14, have reduced proteinase $\mathrm{K}$ resistance which again suggests different conformations of the scrapie and Nor98 prions.

\section{ABSENCE OF DETECTABLE LYMPHOTROPISM IN ATYPICAL/NOR98 SCRAPIE CASES}

In contrast to the BSE strain in cattle, most of the known sheep scrapie strains are lymphotropic, meaning that $\mathrm{PrP}^{\mathrm{Sc}}$ replicate in the lymphoreticular system before neuroinvasion. Several studies have indicated that it might not be the case for the atypical/Nor98 scrapie, where no detectable $\mathrm{PrP}^{\mathrm{Sc}}$ is found in peripheral lymphoid tissues $[5,9,31,41]$. What determines the organ tropism of a prion strain is still unknown, but two hypotheses could be envisaged. The first hypothesis would involve the presence or absence of a possible cofactor in the involved cells that will efficiently or not convert $\mathrm{PrP}^{\mathrm{C}}$ into $\mathrm{PrP}^{\mathrm{Sc}}$. The second hypothesis would be that the tertiary structure of the prion agent itself is, or is not, compatible with the binding to different tissue molecules such as receptors, therefore favouring or not the replication of $\mathrm{PrP}^{\mathrm{Sc}}$ in the lymphoid cells.
Whatever the explanation is, one can speculate that, in the case of the atypical/Nor98 scrapie cases, either the agent is transported directly from the gut to the central nervous system when the TSE agent is ingested, or that the conversion from $\mathrm{PrP}^{\mathrm{C}}$ to $\mathrm{PrP}^{\mathrm{Sc}}$ happens originally in the brain in cases of spontaneous origin.

\section{EPIDEMIOLOGY}

Atypical scrapie Nor98 was first detected in Norway in 1998 and was first considered an isolated Norwegian problem. Due to the suspected presence of BSE in sheep and goats an intensive active surveillance of TSE in small ruminants based on sampling of fallen stock and sheep and goats in normal slaughter by the use of rapid tests was introduced in $2002^{4}$. The intensive active surveillance has led to the detection of atypical/Nor98 scrapie cases in most European countries as well as in the Falkland Islands and in North America [12]. The epidemiology of atypical/Nor98 scrapie is not yet fully understood and it is not established whether it is naturally transmissible or not under natural conditions.

While the majority of the cases have been detected in sheep, a limited number of atypical scrapie cases in goats have been reported from France, Italy ${ }^{5}$, and Switzerland [37]. The findings in the Swiss goat, showing that sampling of the brainstem might not be optimal to detect atypical scrapie in goats, is of epidemiological interest since this might affect the number of cases that are detected [37] but the available epidemiological data on the cases in goats is even more restricted and the following epidemiological information is focussed on data from sheep cases.

\footnotetext{
${ }^{4}$ Regulation (EC) No 999/2001 of the European Parliament and of the Council of 22 May 2001 laying down rules for the prevention, control and eradication of certain transmissible spongiform encephalopathies.

${ }^{5}$ Report on the monitoring and testing of ruminants for the presence of transmissible spongiform encephalopathy (TSE) in the EU in 2005, Luxembourg: Office for Official Publications of the European Communities, 2006, ISBN 92-79-02299-7.
} 


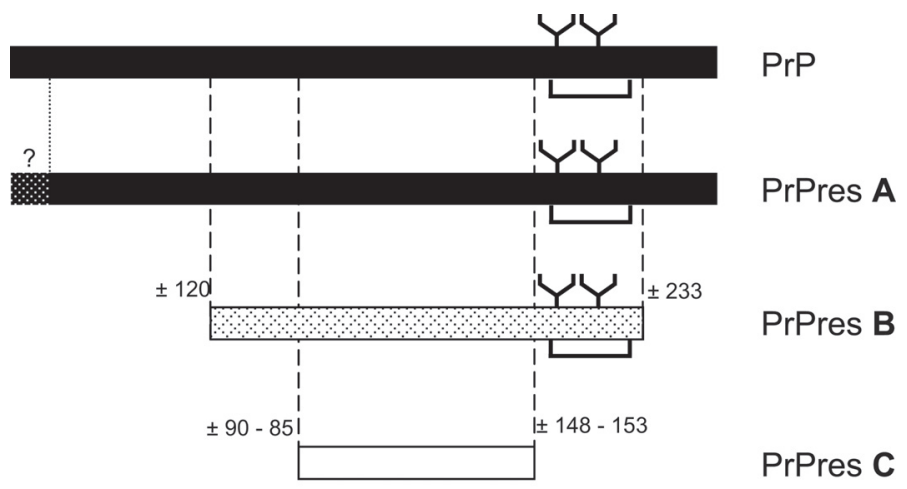

Figure 3. Line diagram illustrating the fragmentation of the atypical/Nor98 PrP.

\subsection{Atypical/Nor98 scrapie animals are older}

The majority of cases have, as mentioned above, been detected through active surveillance by sampling fallen animals and animals at normal slaughter. Active surveillance within the European Union has covered animals above 18 months of age and this is often determined by dentition. In most countries, sheep are not individually identified and year of birth is therefore not always available when a case is detected. For 84 cases in nine different countries where age data was reported, the mean age was 6.5 years $^{6}$. In a German dataset including 60 atypical cases, almost $60 \%$ were above the age of five years, more than $25 \%$ were older than ten years, and cases up to 20 years of age were detected. In the annual TSEreport for 2005, data available on the age of atypical scrapie cases is shown in a graph comparing classical and atypical scrapie where the ages of atypical cases appear to be higher than the classical cases ${ }^{5}$.

\subsection{Prevalence and factors affecting the ability to detect atypical/Nor98 scrapie cases}

The detected prevalence of atypical/Nor98 scrapie cases can be influenced by several factors, which make real comparison between

\footnotetext{
${ }^{6}$ Nöremark M., Hopp P., Reported occurrence of atypical scrapie in Europe, Proceedings of the 11th Symposium of the International Society for Veterinary Epidemiology and Economics, Cairns, Australia: ISVEE 11, 1030, 2006.
}

countries delicate. First, not all tests used for TSE-surveillance in small ruminants will detect atypical/Nor98 scrapie. In a dataset on TSE-surveillance in sheep in Europe from 2002-2005, it was found that 265 of the 294 cases detected were detected by the use of the same rapid test, even though this test only accounted for $42 \%$ of the more than 1.1 million sheep examined ${ }^{6}$. Second, the results of the tests depend on which part of the brain the test is applied to since the amount of $\mathrm{PrP}^{\mathrm{Sc}}$ is different in atypical/Nor98 scrapie cases compared to classical scrapie [16]. Third, the choice of the test for confirmation and the brain region investigated for confirmation will also affect the ability to detect atypical scrapie, since it has been reported that the atypical/Nor98 scrapie cases might be negative by immunohistochemistry at the level of the obex $[5,9,10,16,32]$. And then finally, the population structure (age-structure, predominant genotypes, systems for collecting fallen stock) and the design of the surveillance programme can also have an effect on the detected prevalence. This is of special importance since the atypical/Nor98 scrapie cases appear to be older than the classical scrapie cases.

Some approaches have been made to compare the prevalences between countries. When the rapid test used was taken into account on data from 2004, the detected prevalence of atypical scrapie in the sampled population was quite similar in seven different countries ${ }^{6}$. 
There was a much larger variation when comparing detected prevalence of classical scrapie between these countries ${ }^{7}$. These findings are supported by an extended study performed in 2006-2007 ${ }^{8}$.

Considering geographical aspects, both in Norway and Germany, cases of atypical scrapie have been detected all over the country compared to outbreaks of classical scrapie, which have been concentrated to parts of the country [22,27].

\subsection{Few secondary cases have been reported}

The control measures have varied in different countries, and over time. Stamping out and sampling of all animals in the flock where a positive case has been detected could increase the possibility to detect secondary cases and this has been done in some countries, but the published data on culled animals in relation to detected secondary cases is limited, and reports of detecting only single cases in each flock are common. But there are some reports on flocks where several cases have been detected. In a German comparison of the epidemiology of classical scrapie and atypical scrapie, secondary cases were detected in seven flocks, two of these flocks had more than 500 animals and five of the flocks had more than 1500 animals, but it is not clear how many animals were actually examined in these flocks, in the same study only single cases were detected in 81 of the flocks [27]. Two cases were reported from a flock with 650 sheep in the UK [23]. There is also one report on two cases detected in a small Irish flock [32].

\subsection{Investigation of risk factors}

A study in Great Britain identified that farms with atypical/Nor98 scrapie had significantly higher flock size compared to controlfarms and also a greater number of movements

\footnotetext{
${ }^{7}$ Report on the monitoring and testing of ruminants for the presence of transmissible spongiform encephalopathy (TSE) in 2002, European Commission, Health and consumer protection directorate general, ISBN 92-894-5829-1.

${ }^{8}$ Fediaevsky, Hopp, Nöremark, Tongue, unpublished data.
}

of sheep on and off the farm ${ }^{9}$. A case-control study in Norway on 28 case flocks identified giving vitamin and mineral feed supplement as a risk factor for atypical/Nor98 scrapie, which was different from a previous study on classical scrapie in Norway where various types of contacts with scrapie infected flocks were identified as risk factors $[21,22]$. There was no evidence for association between farms with cases of atypical scrapie, neither in the Norwegian study [22] nor in the Great Britain study ${ }^{9}$.

With regards to the epidemiology of atypical scrapie, many questions still remain to be answered.

Even though some flocks have had several cases this does not imply that atypical scrapie would be contagious. Assuming a spontaneous/sporadic origin, at the detected prevalence in the sampled population in Europe, several cases would be expected in some flocks just by chance related to the size of the flock. Hopp et al. [22] concluded that "Scrapie Nor98 has a low transmissibility or might not be transmitted between animals under natural conditions". Race et al. [35] observed that in the TSE in which lymphoid tissues are extensively involved, i.e., sheep scrapie and CWD in deer, the horizontal transmission in natural conditions is efficient. In contrast, when the peripheral lymphoid tissues are not substantially involved, i.e., BSE in cattle and naturally occurring CWD in elk, the horizontal transmission appears to be relatively inefficient. The restrictive neutropism of the atypical/Nor98 is unexplained, but one could speculate that this characteristic would explain at least partially the low rate of natural transmission between sheep in the atypical/Nor98 scrapie flocks.

The detected prevalence of atypical/Nor98 scrapie in different countries shows a much more homogeneous pattern than what can normally be expected for a contagious disease ${ }^{5,6}$. These findings might support the hypothesis of

\footnotetext{
${ }^{9}$ Green D., del Rio Vilas V., Birch C., Kao R., Patterns of atypical scrapie in Great Britain, in Poster presentation at SVEPM, 28-30 March 2007, Dipoli, Helsinki/Espoo, Finland, http://www.svepm.org.uk/posters/2007/Green.2.pdf [consulted 23 October 2007].
} 
a sporadic, possibly spontaneous, occurrence of atypical/Nor98 scrapie cases, as first raised by Benestad et al. in 2003 [4,5].

\section{EXPERIMENTAL TRANSMISSION RESULTS}

\subsection{Failure of experimental transmission to standard mice}

Experimental transmission studies of atypical/Nor98 scrapie material into standard mice (RIII, VM, and C56Bl mice) have been performed but without succeeding in transmitting the disease within the life span of the mice.

\subsection{Successful experimental transmission to transgenic mice}

The later development of lines of transgenic mice expressing ovine VRQ PrP have given new information about the agent, by efficiently transmitting the disease [25] and therefore proving for the first time that atypical scrapie (also called "discordant" because they were not identified by all the diagnostic tests)/Nor98 was a truly infectious TSE agent.

Furthermore, the neuro-anatomical distribution of $\mathrm{PrP}^{\mathrm{Sc}}$ was analysed by histoblot in discordant and Nor98-infected mice at the terminal stage of the disease to further characterise the TSE agent involved. The $\mathrm{PrP}^{\mathrm{Sc}}$ deposition pattern was clearly distinct from the other analysed TSE isolates, and was remarkably similar for all the discordant/Nor98 isolates, with the preferential involvement of the thalamus, corpus callosum, cerebral cortex, and, to a lesser extent, of the superior colliculus. No $\mathrm{PrP}^{\mathrm{Sc}}$ was detected in the hypothalamus, midbrain, brain stem, or cerebellum. The identical pattern found in all the mice brains infected by discordant/Nor98 isolates (nine sheep and one goat) indicates that the agent involved is closely related, if not identical.

\subsection{Experimental transmission into bank voles}

In an Italian study [7], all the classical scrapie isolates used transmitted efficiently to bank voles with incubation times ranging between 140 and 300 days, but the Nor98 samples, including a sheep that died in the UK in
1989, did not induce clinical disease after first passage within 600 days post inoculation.

\subsection{Successful experimental transmission to sheep}

To the authors' knowledge, only one successful experimental transmission of atypical/Nor98 scrapie into a natural host has yet been reported, in the $\mathrm{UK}^{10}$. An AHQ/AHQ sheep went down with the disease 378 days after intracerebral inoculation with brain material from homologous AHQ/AHQ atypical case. The inoculated sheep showed weight loss, altered behaviour and compulsive circling, and all the neuropathological and molecular characteristics of the donor sheep was preserved upon transmission, including the predominant accumulation of $\mathrm{PrP}^{\mathrm{Sc}}$ immunostaining in the cerebellar, thalamic, basal ganglions, and cortical regions, and its relative absence in the brain stem, and the presence of the distinctive lower band at approximately $12 \mathrm{kDa}$ by Western blot investigation. A second case of successful transmission has been reported by the same research group but has not yet been published.

\section{ASSOCIATION OF PRP GENETICS WITH ATYPICAL SCRAPIE}

Almost a decade after the discovery of atypical/Nor98 scrapie [5], there are still many unsolved questions about the genetic association between PrP and susceptibility. Evidence so far indicates that atypical scrapie affects animals with PrP genotypes different from classical scrapie [2, 3, 9, 26, 27, 29, 30, 33, 36]. The appearance of atypical/Nor98 scrapie and classical scrapie in the same flock has not been reported as a common event, although there is no apparent genetic reason for this: indeed the separation of susceptible genotypes would perfectly permit infection with both diseases in an infected flock. It almost appears as

${ }^{10}$ Simmons M.M., Simmons H.A., Lockey R., Konold T., Spenser Y.I., Spiropoulos J., Clifford D., Phenotype is preserved following experimental transmission of atypical scrapie to sheep, in Abstract book - Conference NeuroPrion 2006 Strategies, advances and trends towards protection of society, 3-6 October 2006, Torino, Italy, p. 219. 
if atypical/Nor98 scrapie is occupying a host niche that is occupied by sheep that are genetically resistant to classical scrapie. Why should that be so? Is this an indicator of competition between strains, an exclusion of double infection? We do not know the answer yet. There is an alternative view that atypical/Nor98 scrapie is a spontaneous (sporadic, non-contagious) disease, because there are in general only individual atypical/Nor98 scrapie cases detected in each flock. Atypical/Nor98 scrapie would not be the first TSE to be classified as such: sporadic CJD (sCJD) in humans also reveals no indication to the origin of disease. Cases of SCJD are unrelated, non-familiar, and have no apparent genetic association. They happen at any place and independent of other TSE [28]. It remains to be established in the coming years by collecting more cases whether the spontaneous disease model will be correct or whether atypical scrapie is an infectious disease like most TSE with an aetiology similar - and maybe complementary - to classical scrapie.

One of the first full descriptions of the PrP genetics for atypical/Nor98 scrapie was provided by Moum et al. [30]. Performing fourcodon genotyping (codons 136, 141, 154, and 171) on 38 cases of Nor98 collected between 1998 and 2004, they came to three major conclusions. Firstly, all animals were of $\mathrm{AA}_{136}$ PrP genotype. The VRQ allele conferring susceptibility to classical scrapie in Norway [40] was completely absent from Nor98 cases although it was present in healthy flock mates. Secondly, there was an over-representation of animals carrying the AHQ allele, in $\mathrm{HH}_{154}$ homozygous and $\mathrm{HR}_{154}$ heterozygous genotypes. Thirdly, the $\mathrm{AF}_{141} \mathrm{RQ}$ allele appeared to confer higher susceptibility than the $\mathrm{AL}_{141} \mathrm{RQ}$ allele. Indeed, the $\mathrm{AF}_{141} \mathrm{RQ}$ allele conferred a higher risk than the AHQ allele. The relative high frequency of the ARR allele in affected heterozygous animals was also surprising. This allele is quite rare in classical scrapie cases. But no ARR/ARR affected sheep were found. The mean age at onset was six years, but no significant association between age and genotype was revealed. It could, however, be possible that this average age, which is higher than that for classical scrapie, may be due to the unusual genotypes that are involved. This hypothesis is nevertheless weakened by the observation that the sheep carrying the ARQ/ARQ PrP genotype, susceptible to both classical and atypical/Nor98 scrapie, are also older when affected by atypical/Nor98 scrapie than by classical scrapie ${ }^{11}$.

There are now additional studies of atypical/Nor98 scrapie from Germany [27], France [2], and the UK [7, 36], all pointing to the same conclusion, that this type of scrapie is strongly associated with PrP codons 141 and 154 (Tab. II). Importantly, they described ARR/ARR carriers with atypical scrapie $[2,9,27,33,36]$. The susceptibility of $\mathrm{RR}_{171}$ homozygotes has been a major concern because national breeding and eradication plans for classical scrapie in EU member states are inadvertently creating a high frequency of ARR/ARR animals. The studies also proved that $\mathrm{VA}_{136}$ heterozygotes were susceptible to atypical/Nor98 scrapie [2,36] but this appears to be linked to the presence of the $\mathrm{AF}_{141} \mathrm{RQ}$ allele in these animals.

Atypical/Nor98 scrapie is also found in goats. Like in sheep, goats with $\mathrm{HH}_{154}$ and $\mathrm{HR}_{154}$ PrP genotypes appear to be targeted by this atypical/Nor98 scrapie [2], although it is too early to confirm the association statistically.

Experimental challenges of sheep and ovine PrP transgenic mice with atypical/Nor98 scrapie sources are essential tools for the future to decipher the true extent of susceptible genotypes. We may then also be able to explain why the VRQ/AHQ genotype has a low risk for both types of scrapie, although it is composed of the two alleles with highest susceptibility risk for classical [3] and atypical/Nor98 [2, 27, 30, 36] scrapie, respectively. The discovery of atypical/Nor98 scrapie has opened up a new phase in the exploration of the link between the PrP gene and disease, which in the end is likely to contribute fundamentally to the understanding of the underlying molecular mechanisms.

\footnotetext{
${ }^{11}$ Norwegian data, Benestad, Bratberg, Hopp, unpublished data.
} 
Table II. Compilation of genotypes from 241 cases of atypical/Nor98 scrapie from four countries [2, 27,30, 36].

\begin{tabular}{lcc}
\hline Genotype (136/141/154/171) & Observations $(n=241)$ & Frequency $(\%)$ \\
\hline ALRQ/ALHQ & 34 & 14.1 \\
ALHQ/ALRR & 32 & 13.3 \\
AFRQ/ALRR & 31 & 12.9 \\
ALHQ/ALHQ & 29 & 12.0 \\
AFRQ/AFRQ & 23 & 9.5 \\
AFRQ/ALRQ & 23 & 9.5 \\
AFRQ/ALHQ & 21 & 8.7 \\
ALRR/ALRR & 21 & 8.7 \\
ALRQ/ALRQ & 8 & 3.3 \\
ALRQ/ALRR & 8 & 3.3 \\
AFRQ/VLRQ & 5 & 2.1 \\
ALHQ/ALRH & 3 & 1.3 \\
ALHQ/VLRQ & 1 & 0.4 \\
ALRH/ALRR & 1 & 0.4 \\
ALRH/ALRH & 1 & 0.4 \\
AFRQ/ALRH & 0 & 0 \\
ALRQ/ALRH & 0 & 0 \\
ALRQ/VLRQ & 0 & 0 \\
ALRH/VLRQ & 0 & 0 \\
ALRR/VLRQ & 0 & 0 \\
VLRQ/VLRQ & 0 & 0 \\
\hline
\end{tabular}

\section{BIO-DIVERSITY OR UNIFORMITY OF ATYPICAL/NOR98 SCRAPIE ISOLATES?}

Arsac et al. [2] demonstrated that the isolates from 54 French atypical cases which included a large panel of different PrP genotypes and two species (sheep and goat) showed identical glycoprofile of $\mathrm{PrP}^{\mathrm{Sc}}$ were examined and were shown to possess a unique biochemical signature indistinguishable from that of the Norwegian Nor98 isolates. In addition, Le Dur et al. [25] showed that all the isolates from the French atypical ("discordant") and Norwegian Nor98 cases (nine sheep and one goat) that were transmitted to $\operatorname{tg} 338$ ovine mice also shared the same biological signature with comparable incubation durations, clinical sign lesion profiles, $\mathrm{PrP}^{\mathrm{Sc}}$ distribution patterns in the brain and the apparent lack of tropism for the lymphoreticular tissues.

These results suggest that the prions involved in atypical and Nor98 cases are in fact a unique TSE agent. It should nevertheless be noted that only a small number of cases that has been investigated, originating from only two countries (France and Norway), and a real diversity in the group of atypical scrapie/Nor98 cases might possibly be observed if additional isolates were investigated under the same experimental diagnostic conditions. It can therefore not be excluded that atypical scrapie/Nor98 might be a heterogeneous group that could be further divided into different subtypes, but the attempt to group them in separate entities based on consistent strain characteristics/features have not yet succeeded.

\section{CONCLUSION}

Initially atypical/Nor98 scrapie was thought to be another type of classical scrapie but has now been identified to be a strain or a group of TSE affecting small ruminants which is clearly distinguishable from classical scrapie and from BSE in sheep ${ }^{2}$ [8]. The intensive TSE surveillance within Europe has shown that the disease is quite spread and often occurring as single cases in a flock. The epidemiological data accumulated during recent years have also led to a separation of classical scrapie and atypical/Nor98 scrapie with 
regards to control measures applied in positive flocks within the European Union ${ }^{4}$.

Despite the fact that this strain is now better characterised, its origin is still unknown and further transmission studies, as well as collection and analysis of epidemiological data from the field, are needed to give information about the pathogenesis of this strain to fully understand the epidemiology and to assess the possible zoonotic aspects.

Acknowledgements. Work in the authors' laboratories was supported by the NeuroPrion European Network of Excellence, grants from the Norwegian Research Council (146916/140 and 161748/110) and from Biotechnology and Biological Sciences Research Council UK.

\section{REFERENCES}

[1] Aguzzi A., Heikenwalder M., Polymenidou M., Insights into prion strains and neurotoxicity, Nat. Rev. Mol. Cell Biol. (2007) 8:552-561.

[2] Arsac J.N., Andréoletti O., Bilheude J.M., Lacroux C., Benestad S.L., Baron T., Similar biochemical signatures and prion protein genotypes in atypical scrapie and Nor98 cases, France and Norway, Emerging Infect. Dis. (2007) 13:58-65.

[3] Baylis M., Goldmann W., The genetics of scrapie in sheep and goats, Curr. Mol. Med. (2004) 4: 385-396.

[4] Baron T., Biacabe A.G., Arsac J.N., Benestad S., Groschup M.H., Atypical transmissible spongiform encephalopathies (TSEs) in ruminants, Vaccine (2007) 25:5625-5630.

[5] Benestad S.L., Sarradin P., Thu B., Schönheit J., Tranulis M.A., Bratberg B., Cases of scrapie with unusual features in Norway and designation of a new type, Nor98, Vet. Rec. (2003) 153:202-208.

[6] Benestad S.L., Bratberg B., Atypical scrapieNor98, in: Beat Hörnlimann, Detlev Riesner, Hans Kretzschmar (Eds), Prions in Humans and Animals, de Gruyter, Berlin, Germany, 2006, pp. 630-634.

[7] Bruce M.E., Nonno R., Foster J., Goldmann W., Di Bari M., Esposito E., Benestad S.L., Hunter N., Agrimi U., Nor98-like sheep scrapie in the United Kingdom in 1989, Vet. Rec. (2007) 160:665-666.

[8] Buschmann A., Groschup M.H., TSE eradication in small ruminants - quo vadis?, Berl. Munch. Tierarztl. Wochenschr. (2005) 118:365-371.

[9] Buschmann A., Biacabe A.G., Ziegler U., Bencsik A., Madec J.Y., Erhardt G., Lühken G., Baron T., Groschup M.H., Atypical scrapie cases in Germany and France are identified by discrepant reaction patterns in BSE rapid tests, J. Virol. Methods (2004) 117:27-36.
[10] Buschmann A., Lühken G., Schultz J., Erhardt G., Groschup M.H., Neuronal accumulation of abnormal prion protein in sheep carrying a scrapieresistant genotype (PrPARR/ARR), J. Gen. Virol. (2004) 85:2727-2733.

[11] Collinge J., Sidle K.C., Meads J., Ironside J., Hill A.F., Molecular analysis of prion strain variation and the aetiology of "new variant" CJD, Nature (1996) 383:685-690.

[12] Cook W., Nor98-like strain of scrapie found in Wyoming, Wyoming Livestock Board (2007).

[13] De Bosschere H., Roels S., Benestad S.L., Vanopdenbosch E., Scrapie case similar to Nor98 diagnosed in Belgium via active surveillance, Vet. Rec. (2004) 155:707-708.

[14] Epstein V., Pointing S., Halfacre S., Atypical scrapie in the Falkland Islands, Vet. Rec. (2005) 157:667-668.

[15] Everest S.J., Thorne L., Barnicle D.A., Edwards J.C., Elliott H., Jackman R., Hope J., Atypical prion protein in sheep brain collected during the British scrapie-surveillance programme, J. Gen. Virol. (2006) 87:471-477.

[16] Gavier-Widén D., Nöremark M., Benestad S., Simmons M., Renström L., Bratberg B., Elvander M., af Segerstad C.H., Recognition of the Nor98 variant of scrapie in the Swedish sheep population, J. Vet. Diagn. Invest. (2004) 16:562-567.

[17] González L., Martin S., Jeffrey M., Distinct profiles of $\operatorname{PrP}(\mathrm{d})$ immunoreactivity in the brain of scrapie- and BSE-infected sheep: implications for differential cell targeting and PrP processing, J. Gen. Virol. (2003) 84:1339-1350.

[18] Gretzschel A., Buschmann A., Langeveld J., Groschup M.H., Immunological characterization of abnormal prion protein from atypical scrapie cases in sheep using a panel of monoclonal antibodies, J. Gen. Virol. (2006) 87:3715-3722.

[19] Hayashi H.K., Yokoyama T., Takata M., Iwamaru Y., Imamura M., Ushiki Y.K., Shinagawa M., The Nterminal cleavage site of PrPSc from BSE differs from that of PrPSc from scrapie, Biochem. Biophys. Res. Commun. (2005) 328:1024-1027.

[20] Hope J., Wood S.C., Birkett C.R., Chong A., Bruce M.E., Cairns D., Goldmann W., Hunter N., Bostock C.J., Molecular analysis of ovine prion protein identifies similarities between BSE and an experimental isolate of natural scrapie, J. Gen. Virol. (1999) 80:1-4.

[21] Hopp P., Ulvund M.J., Jarp J., A case-control study on scrapie in Norwegian sheep flocks, Prev. Vet. Med. (2001) 51:183-198.

[22] Hopp P., Omer M.K., Heier B.T., A case-control study of scrapie Nor98 in Norwegian sheep flocks, J. Gen. Virol. (2006) 87:3729-3736.

[23] Konold T., Davis A., Bone G., Bracegirdle J., Everitt S., Chaplin M., Saunders G.C., Cawthraw S., 
Simmons M.M., Clinical findings in two cases of atypical scrapie in sheep: a case report, BMC Vet. Res. (2007) 3:2.

[24] Klingeborn M., Wik L., Simonsson M., Renström L.H., Ottinger T., Linne T., Characterization of proteinase K-resistant $\mathrm{N}$ - and C-terminally truncated PrP in Nor98 atypical scrapie, J. Gen. Virol. (2006) 87:1751-1760.

[25] Le Dur A., Béringue V., Andréoletti O., Reine F., Laï T.L., Baron T., Bratberg B., Vilotte J.L., Sarradin P., Benestad S.L., Laude H., A newly identified type of scrapie agent can naturally infect sheep with resistant PrP genotypes, Proc. Natl. Acad. Sci. USA (2005) 102:16031-16036.

[26] Lühken G., Buschmann A., Groschup M.H., Erhardt G., Prion protein allele A136 H154Q171 is associated with high susceptibility to scrapie in purebred and crossbred German Merinoland sheep, Arch. Virol. (2004) 149:1571-1580.

[27] Lühken G., Buschmann A., Brandt H., Eiden M., Groschup M.H., Erhardt G., Epidemiological and genetical differences between classical and atypical scrapie cases, Vet. Res. (2007) 38:65-80.

[28] Mead S., Prion disease genetics, Eur. J. Hum. Genet. (2006) 14:273-281.

[29] Moreno C.R., Moazami-Goudarzi K., Laurent P., Cazeau G., Andréoletti O., Chadi S., Elsen J.M., Calavas D., Which PrP haplotypes in a French sheep population are the most susceptible to atypical scrapie? Arch. Virol. (2007) 152:1229-1232.

[30] Moum T., Olsaker I., Hopp P., Moldal T., Valheim M., Moum T., Benestad S.L., Polymorphisms at codons 141 and 154 in the ovine prion protein gene are associated with scrapie Nor98 cases, J. Gen. Virol. (2005) 86:231-235.

[31] Nentwig A., Oevermann A., Heim D., Botteron C., Zellweger K., Drögemüller C., Zurbriggen A., Seuberlich T., Diversity in neuroanatomical distribution of abnormal prion protein in atypical scrapie, PLoS Pathog. (2007) 3:e82.
[32] Onnasch H., Gunn H.M., Bradshaw B.J., Benestad S.L., Bassett H.F., Two Irish cases of scrapie resembling Nor98, Vet. Rec. (2004) 155:636-637.

[33] Orge L., Galo A., Machado C., Lima C., Ochoa C., Silva J., Ramos M., Simas J.P., Identification of putative atypical scrapie in sheep in Portugal, J. Gen. Virol. (2004) 85:3487-3491.

[34] Prusiner S.B., Novel proteinaceous infectious particles cause scrapie, Science (1982) 216:136-144.

[35] Race B.L., Meade-White K.D., Ward A., Jewell J., Miller M.W., Williams E.S., Chesebro B., Race R.E., Levels of abnormal prion protein in deer and elk with chronic wasting disease, Emerging Infect. Dis. (2007) 13:824-830.

[36] Saunders G.C., Cawthraw S., Mountjoy S.J., Hope J., Windl O., PrP genotypes of atypical scrapie cases in Great Britain, J. Gen. Virol. (2006) 87:31413149.

[37] Seuberlich T., Botteron C., Benestad S.L., Brünisholz H., Wyss R., Kihm U., Schwermer H., Friess M., Nicolier A., Heim D., Zurbriggen A., Atypical scrapie in a Swiss goat and implications for transmissible spongiform encephalopathy surveillance, J. Vet. Diagn. Invest. (2007) 19:2-8.

[38] Somerville R.A., Chong A., Mulqueen O.U., Birkett C.R., Wood S.C., Hope J., Biochemical typing of scrapie strains, Nature (1997) 386:564.

[39] Stockman S., Scrapie: an obscure disease in sheep, J. Comp. Pathol. (1913) 26:317-327.

[40] Tranulis M.A., Osland A., Bratberg B., Ulvund M.J., Prion protein gene polymorphisms in sheep with natural scrapie and healthy controls in Norway, J. Gen. Virol (1999) 80:1073-1077.

[41] Vidal E., Tortosa R., Costa C., Benavides J., Francino O., Sánchez-Robert E., Pérez V., Pumarola M., Lack of $\operatorname{PrP}(\mathrm{Sc})$ immunostaining in intracranial ectopic lymphoid follicles in a sheep with concomitant non-suppurative encephalitis and Nor98-like atypical scrapie: a case report, Vet. J. (2007) DOI: 10.1016/j. tvj1. 2007.04.014. 